\title{
PROMIS-29 Adult Profile v1.0
}

National Cancer Institute

\section{Source}

National Cancer Institute. PROMIS-29 Adult Profile v1.0. NCI Thesaurus. Code C132903.

A 29-item multidimensional and universal measure of health intended for use across a variety of conditions. It measures an adult patient's self-reported health status in seven domains: physical function, anxiety, depression, fatigue, sleep disturbance, pain interference, and participation in social roles. 